\title{
Analysis of hybrid subcarrier multiplexing of OCDMA based on single photodiode detection
}

\author{
N. A. A Ahmad ${ }^{1, *}$, M. N Junita ${ }^{1}$, S.A. Aljunid ${ }^{1}$, C.B.M. Rashidi ${ }^{1}$, and R. Endut ${ }^{1}$ \\ ${ }^{1}$ Advanced Communication Engineering, Centre of Excellence, School of Computer and Communication Engineering, Universiti \\ Malaysia Perlis, Arau, 01000, Perlis, Malaysia.
}

\begin{abstract}
This paper analyzes the performance of subcarrier multiplexing (SCM) of spectral amplitude coding optical code multiple access (SAC-OCDMA) by applying Recursive Combinatorial (RC) code based on single photodiode detection (SPD). SPD is used in the receiver part to reduce the effect of multiple access interference (MAI) which contributes as a dominant noise in incoherent SAC-OCDMA systems. Results indicate that the SCM OCDMA network performance could be improved by using lower data rates and higher number of weight. Total number of users can also be enhanced by adding lower data rates and higher number of subcarriers.
\end{abstract}

\section{Introduction}

Recently, researchers have shown an increased interest in optical code division multiple access (OCDMA). OCDMA is an optical-based multiple-access method which can transmit the data simultaneously from several users over the network and can be distinguished by its signature of the code based on specific detection. In addition, OCDMA scheme is good in combining the unlimited bandwidth of fiber with the flexibility of CDMA technique to accomplish high-quality transmission without overwhelming the traffic management or system synchronization [1]. Besides that, OCDMA also have numerous advantages. For instance, it provides secure transmission, ability to support asynchronous access network, does not need network protocol and affordable cost [2]. However, OCDMA also have some disadvantages. The major drawback of OCDMA is, the performance of the system degrades owing to the effects of multiple access interference (MAI) [3].

Thereby, Spectral amplitude coding (SAC) approaches have been proposed. It is a flexible implementation of optical access networks for long-haul transmission. It gives a better quality transmission with capability to reduce the effects of multiple access interference (MAI). Moreover, for synchronization, no electronic are needed. Then, it offers a low cost, all optical processing, simplified network control, and bandwidth efficiency. An effective SAC-OCDMA system can be achieved by choosing efficient codes for encoding the source bits. In order to select a family code with effectively, it needs to distinguished based on their features such as code length $(\mathrm{N})$, cross-correlation, code weight (w) and number of users (K) [4]. In this work, we utilize Recursive Combinatorial (RC) code because it has minimum code lengths, minimum cross correlation which is equal to one and its flexibility in choosing code parameter [5].

Subcarrier multiplexing (SCM) technique has become increasing demand in OCDMA system since it gives the independency of different channels [6]. This allows for great flexibility in the choice of modulation schemes. There are increasing demands for high data rates with low propagation loss, so the best solution to fulfil the request is by combine subcarrier multiplexed with optical code division multiple accesses to the fiber optic communication system. SCM method becomes high attention because of their advantages. For instance, it able to operate asynchronously, increase in privacy, and increase its capacity when in bursty network[3]. In addition, it is flexible in the allocation of channels.

However, to reduce the effects of MAI, single photodiode detection (SPD) is applied in the SACOCDMA system. SPD detection is proposed because it offers cost-effective and less system complexity compared with other types of SAC detection techniques. The rest of the paper is organized as follows: In Section 2 , we introduce system design based on SPD detection technique. In Section 3, we analyse the results for hybrid SCM OCDMA system based SPD technique. Finally, the conclusion is given in Section 4.

\footnotetext{
*Corresponding author: n.amirahamniahmad@gmail.com
} 


\section{Single Photodiode Detection (SPD)}

In OCDMA systems, the detection schemes affect the design of transmitter and receivers. The types of detection are divided by two which are coherent system and incoherent system. Incoherent system is unipolar sequence for the code sequence whereas coherent system is bipolar sequence for the code words. In our system, we applied incoherent system since the coherent system is phase sensitive, which would be practically difficult to implement in this system.

Fig. 1 illustrates the implementation of SCM OCDMA structure at the transmitter part. Here, data from users are modulated into electrical signal within different specific frequencies. These RF frequencies are then combined using electrical adder before being modulated into optical signal codes via a Mach Zender Modulator. The optical RC codes are executed by splitting the broadband optical sources into smaller wavelengths, and encoded it back in the form of codes using an encoder. Optical combiner is then used to combine the optical codes before being transmitted via a single mode fiber.

Fig. 2 shows the implementation of SPD detection at the receiver. The structure is simple compared to other detections, such as AND detection. It only needs a couple of decoder and photo detector rather than AND subtraction detection where it requires two pairs of decoder and photo detector. After optical subtraction, the output is either code weight power unit for active user or zero power units for interferers. This implies that the interference signals are suppressed in the optical domain before the conversion of the signals to the electrical domain, as a result, the proposed SPD scheme alleviates both PIIN and MAI in the optical domain. Moreover, the two interference signals at the optical subtractor are assumed to be equal and cancel each other out. However, practically, the interference signals differ slightly at the optical subtractor and results in a small amount of optical power to reach the photodiode.

At the photodetector, the photo diode only detects and filters the clean chips or the non-overlapping chips. Then, the effects of MAI are successfully reduced in the optical domain because there are only the required signal spectra are filtered. Then, there is no subtraction process in SPD technique. It is because; the photo detector only detects the clean optical pulses and converts it into electrical domain. The electrically modulated signal is then passed through a Band Pass Filter (BPF) to remove the unwanted frequency. In order to remove the higher frequency, a low pass filter (LPF) is assigned. The original data signal is detected at the output of LPF using a BER analyser.

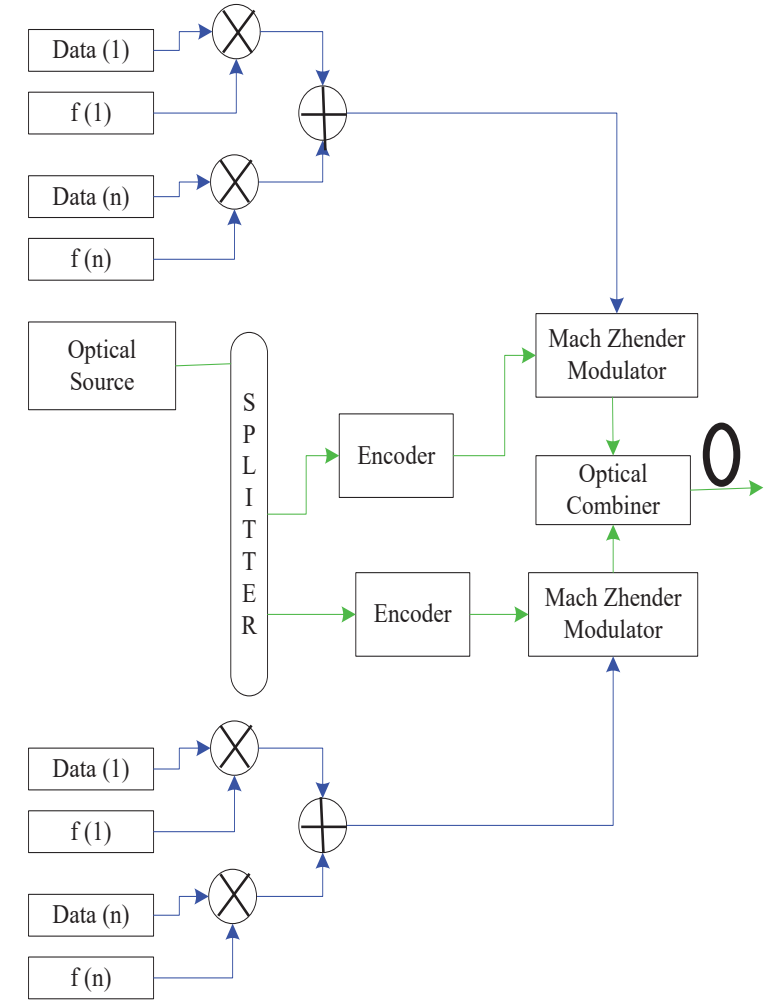

Fig. 1. Transmitter part of hybrid SCM SAC- OCDMA system

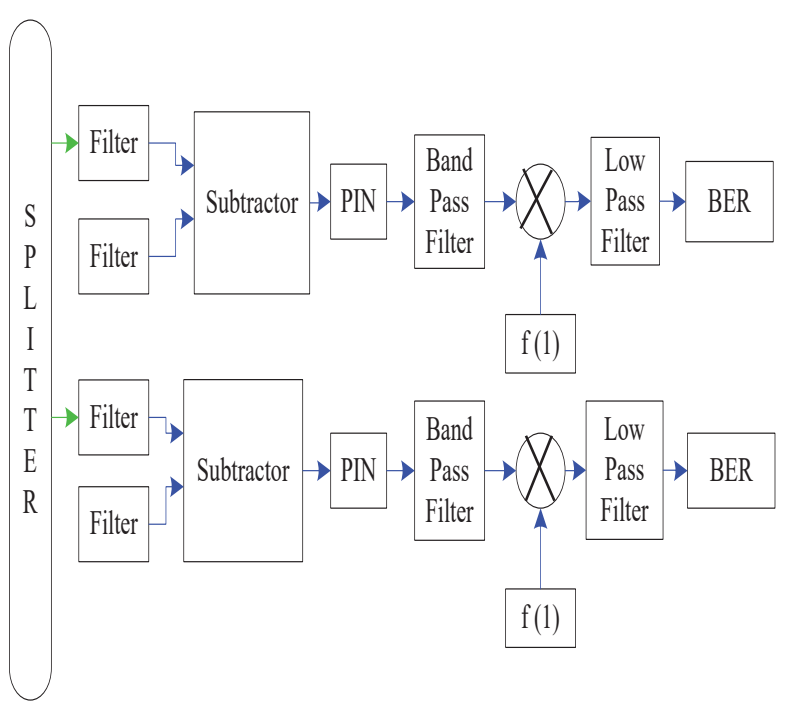

Fig. 2. The receiver part hybrid SCM SAC-OCDMA system based on SPD detection technique 


\section{Theoretical Development of Hybrid SCM OCDMA System Based on SPD Technique}

The performance of RC code in hybrid system can be analysed by describing the properties of RC code which are the cross correlation and auto correlation function of the code take into account. In equation $1, \operatorname{Cd}(i)$ represents the $i$ th element of $A$ th $\mathrm{RC}$ code sequence. So that, the properties of RC code based on SPD method can be illustrated as:

$\sum_{i=1}^{L} C_{d}(i) C_{e}(i)=\left\{\begin{array}{l}W ; \text { for } d=e \\ 1 ; \text { for } d \neq e\end{array}\right.$

Where $\mathrm{C}_{d}$ and $\mathrm{C}_{e}$ depict the $\mathrm{RC}$ code sequences, meanwhile $\mathrm{L}$ depicts the code length and $\mathrm{W}$ depicts the number of the weight. At the receiver part, the photo detector only detects and decodes the non-overlapping chips. Therefore, the total incident power during one period at the input of the photo detector PIN 1 for the SPD detection technique can be expressed by:

$\int_{0}^{\infty} G_{d d}(v) d v=$

$\int_{0}^{\infty} \frac{P_{s r}}{\Delta v} \sum_{k=1}^{K} d_{k} \sum_{i=1}^{L} C_{d}(i) C_{e}(i) u\left[\frac{\Delta v}{L}\right] d v$

$=\frac{P_{s r}}{\Delta v}\left[\frac{\Delta v}{L}\right] \sum_{k=1}^{K} d_{k} \sum_{i=1}^{l} C_{d}(i) C_{e}(i)$

The photodiode current, I indicate the signal from the desired user and can be displays as in eq (3):

$I=I_{d d}=\Re \int_{0}^{\infty} G_{d d}(v) d v$

Then, substitute the equation (4) in (5), the equation becomes:

$I_{d d}=\mathfrak{R} \frac{P_{s r}}{L}(\boldsymbol{w}-\mathbf{1})$

Based on equation (6), (W-1) represents the power spectral density of the overlapping chips or cross correlation are subtracted at the received power signal. Thereby, the photodiode only detects and filters the PSD of the non-overlapping chips.

For the noise variances, there are three types of considered noises which are shot noise, thermal noise and intermodulation noise while PIIN noise is neglected. The PIIN noise is neglected because it only appears when the optical signal is incident more than one on the photo detector surface. The shot noise can be expressed as:

$$
\begin{aligned}
& I_{\text {shot }}^{2}=2 e B I_{d d} \\
& I_{\text {shot }}^{2}=\mathbf{2 e B} \Re \frac{\boldsymbol{P}_{\text {sr }}(\boldsymbol{w}-\mathbf{1})}{\boldsymbol{L}}
\end{aligned}
$$

The thermal noise [3] can be expressed as:

$$
I_{\text {thermal }}^{2}=\frac{4 \mathrm{~K}_{\mathrm{b}} \mathrm{T}_{\mathrm{N}} \mathrm{B}}{\mathrm{R}_{\mathrm{L}}}
$$

The inter-modulation noise [8] can be expressed as:

$$
I_{I M D}^{2}=\mathbf{P}_{\mathrm{sr}}^{2} \Re^{2} \mathbf{m}_{\mathrm{n}, \mathrm{k}}^{6}\left[\frac{\mathrm{D}_{111}}{32}+\frac{\mathrm{D}_{21}}{64}\right]
$$

The Signal Noise Ratio or SNR equation of the hybrid SCM OCDMA using RC code using SPD method can be expressed as:

$S N R=\frac{\left(\frac{\Re P_{s r}(W-1)}{L}\right)^{2} m_{n, k}^{2}}{e B \Re \frac{P_{s r}(W-1)}{L}+\frac{4 K_{b} T_{n} B}{R_{L}}+P_{s r}^{2} \Re^{2} m_{n, k}^{6}\left[\frac{D_{11}}{32}+\frac{D_{21}}{64}\right]}$

Based on equation (10), the formula to calculate the bit error rate (BER) can be written as:

$$
\begin{aligned}
& \mathrm{BER}=\frac{1}{2} \operatorname{erf} \boldsymbol{c}\left(\sqrt{\frac{S N R}{8}}\right) \\
& \text { Here, } \operatorname{erfc}=\frac{2}{\sqrt{\pi}} \int_{x}^{\infty} \exp \left(-z^{2}\right) d z
\end{aligned}
$$

Table 1. List of parameter used in the analysis

\begin{tabular}{|c|c|l|}
\hline Symbol & Parameter & Value \\
\hline $\boldsymbol{\eta}$ & $\begin{array}{c}\text { The efficiency of } \\
\text { photodetector quantum }\end{array}$ & 0.6 \\
\hline$\Delta \boldsymbol{v}$ & $\begin{array}{c}\text { The source of Linewidth } \\
\text { broadband }\end{array}$ & $3.75 \mathrm{THz}$ \\
\hline $\boldsymbol{\lambda}_{\mathbf{0}}$ & $\begin{array}{c}\text { The wavelength of } \\
\text { operating }\end{array}$ & $1550 \mathrm{~nm}$ \\
\hline $\mathrm{B}$ & Electrical bandwidth & $80 / 331 \mathrm{MHz}$ \\
\hline $\mathrm{R}_{\mathrm{b}}$ & Data Bit Rate & $155 / 622 \mathrm{Mbps}$ \\
\hline $\mathrm{T}_{\mathrm{n}}$ & $\begin{array}{c}\text { The temperature of } \\
\text { Receiver noise }\end{array}$ & $300 \mathrm{~K}$ \\
\hline $\mathrm{R}_{\mathrm{L}}$ & Receiver load resistor & $1030 \Omega$ \\
\hline $\mathrm{e}$ & Electron charge & $1.6 \times 10^{-19} \mathrm{C}$ \\
\hline $\mathrm{k}_{\mathrm{b}}$ & Boltzmann's constant & $\begin{array}{l}1.38 \quad \mathrm{x} \quad 10^{-23} \\
\mathrm{~J} / \mathrm{K}\end{array}$ \\
\hline
\end{tabular}




\section{Analysis of Results and Discussion}

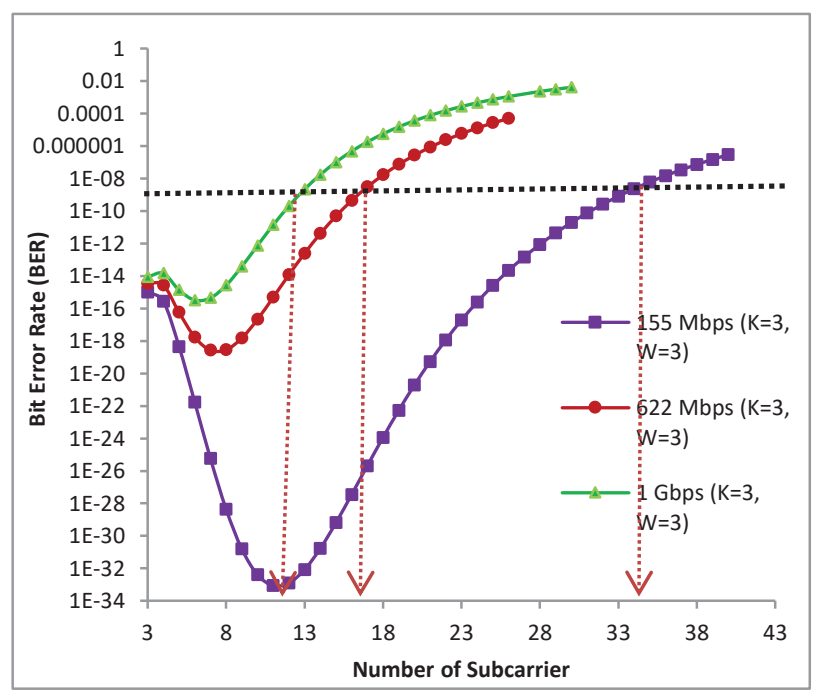

Fig. 3. Bit Error Rates versus number of subcarrier with respect to the data rate

Fig. 3 depicts the relationship between the bit error rate and number of subcarriers with respect to various numbers of data rates such as $155 \mathrm{Mbps}, 622 \mathrm{Mbps}$ and 1 Gbps- As can be seen in Figure 3, when lower data rates are assigned in the system, it can occupy large number of subcarriers. For instance, at the threshold BER value of $10^{-9}$, a system with $155 \mathrm{Mbps}$ per sub channel can accommodate about 35 subcarriers. As data rate increases to $622 \mathrm{Mbps}$, only 17 subcarriers can be hold in one system meanwhile at data rate $1 \mathrm{Gbps}$, only 12 subcarriers can be included in the same system. The system capacity will increase if the numbers of subcarriers increase and the value of bit rate decrease rather than having less number of subcarriers but with higher bit rates. Thereby, the performance of hybrid SCM system can be maintained above threshold BER value $10^{-9}$.

Fig. 4 presents the performance of hybrid SCM system by using different bit rates per sub channel with varying effective transmitted power. As can be seen in Figure 4, the performance of BER for bit rate $155 \mathrm{Mbps}$ is better compared to bit rate $622 \mathrm{Mbps}$. At the BER value $10^{-9}$, the effective power for bit rate $155 \mathrm{Mbps}$ is about -18 $\mathrm{dBm}$ while for the bit rate $622 \mathrm{Mbps}$, the effective power is about $-14 \mathrm{dBm}$ for the same system. Increasing in data rates will increase the electrical bandwidth, then it will degrade the signal to noise ratio. Thus, the performance of the hybrid SCM system will degrade as the data rate increase. As a conclusion lower data rates will increase the system performance.

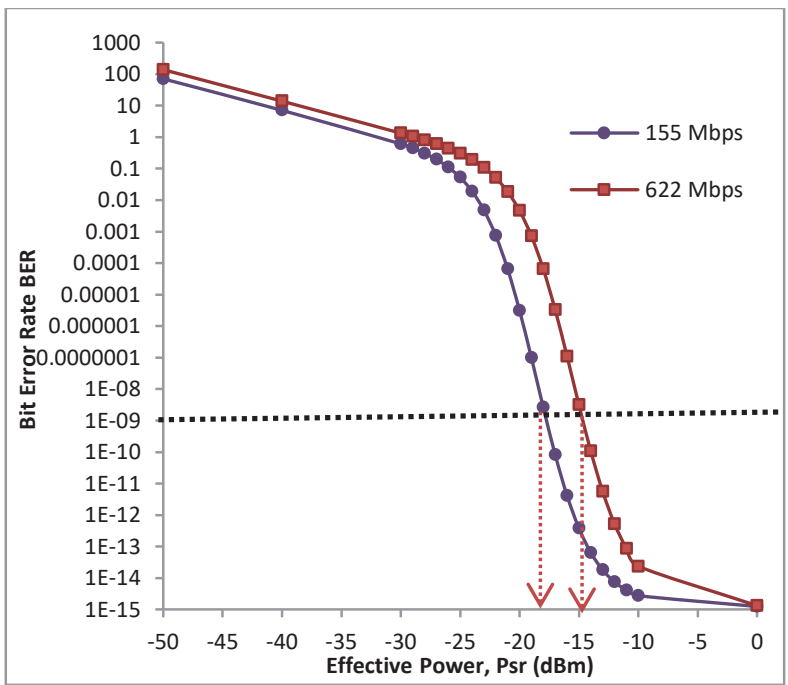

Fig. 4. Bit Error Rates versus Effective Power with respect to bit rates

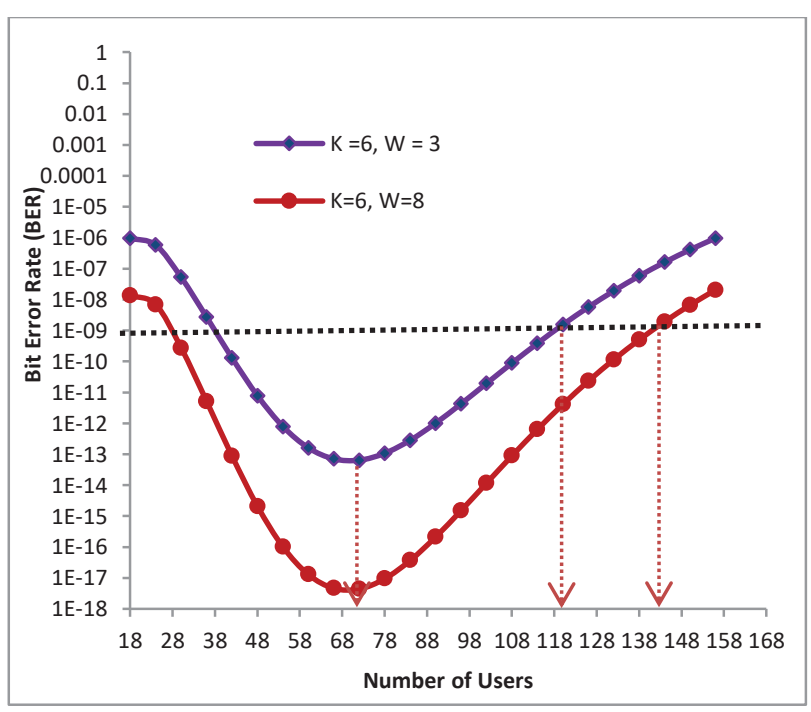

Fig. 5. Bit Error Rates versus Number of Users with respect to number of weight

Fig. 5 depicts the performance of hybrid SCM system with various numbers of weights. The number of optical codes is fixed to six, meanwhile the number of subcarrier is varied. By multiplying the number of code sequence with the number of subcarriers channels, we will get the total number of users. The definition number of weight in OCDMA system is the number of "ones" in code sequences will represent the number of weights. As shown in Figure 5, at BER $10^{-9}$, for the weight three, the total number of users is 120 whereas for the weight eight, the total number of users is 143. A part from that, the optimum BER for width three and width eight are 70 users. Thereby, increasing the number of weight will increase the information-bearing signal power and will enhance the performance of hybrid system. 


\section{CONCLUSION}

This paper presents the hybrid SCM system by implementing SPD technique. The SPD technique have been proposed and analysed to evaluate the performance of the hybrid SCM system by utilized Recursive Combinatorial (RC) code. Based on the results, it can be concluded that, the performance of hybrid system can be increased by reducing the bit rates and increasing the number of weight. As the bit rate decreases, the bandwidth of the system decreases, then the performance will increase. SPD detection technique gives better performance for hybrid system as it reduces the effect of MAI at the optical domain. Thus, it can support higher number of users by increasing the number of subcarrier channel without affecting the number of code lengths and optical codes. In addition, its bandwidth becomes more efficient.

The authors would like to thank the Ministry of Higher Education (MOHE) Malaysia for the funding under the Fundamental Research Grant Scheme (FRGS).

\section{REFERENCES}

1. S. G. Abdulqader, H. A. Fadhil, and S. A. Aljunid. J. Theor. Appl. Inf. Technol., 67(2), pp. 368-372, (2014).

2. H. M. R. Al-Khafaji, S. A. Aljunid, A. Amphawan, H. A. Fadhil, and A. M. Safar. J. Eur. Opt. Soc., vol. 8, (2013).

3. Somaya A. Abd E, Mottaleb, Heba A. Fayed, Ahmed Abd El Aziz, and Moustafa H.Aly. IOSR J. Electron. Commun. Eng., vol. 9(2), pp. 55-60, (2014).

4. M. Z. Norazimah, H. M. R. Al-Khafaji, S. A. Aljunid, and H. A. Fadhil. ICP 2012 - 3rd Int. Conf. Photonics 2012, Proc., no. October, pp. 199-203,( 2012).

5. J. M. Nordin, S. A. Aljunid, A. M. Safar, A. R. Arief, R. A. Rahim, and R. B. Ahmad. Aust. J. Basic Appl. Sci., vol. 8, no. 1, pp. 343-350, (2014).

6. R.K.Z. Sahbudin, M.K. Abdullah, M.D.A. Samad, M.A. Mahdi and M. Ismail. J. of Applied. Sci,vol 7, pp.1942-1947, (2007)

7. S. G. Abdulqader, S. A. Aljunid, H. M. R. Alkhafaji, and H. A. Fadhil. J. Commun., vol. 9(2), pp. 99-106,( 2014).

8. T. H. Abd, S. A. Aljunid, H. A. Fadhil, I. F. Radhi, R. B. Ahmad, and M. A. Rashid. Sci. Res. Essays, vol. 7(11), pp. 1262-1272, (2012). 